Multiple electron cyclotron power deposition location tracking by break-in-slope analysis in TCV plasmas

This article has been downloaded from IOPscience. Please scroll down to see the full text article.

2011 Plasma Phys. Control. Fusion 53115005

(http://iopscience.iop.org/0741-3335/53/11/115005)

View the table of contents for this issue, or go to the journal homepage for more

Download details:

IP Address: 128.178.254.23

The article was downloaded on 10/10/2011 at 10:27

Please note that terms and conditions apply. 


\title{
Multiple electron cyclotron power deposition location tracking by break-in-slope analysis in TCV plasmas
}

\author{
L Curchod ${ }^{1}$, F Felici ${ }^{1}$, A Pochelon ${ }^{1}$, J Decker ${ }^{2}$, T P Goodman ${ }^{1}$, \\ J-M Moret ${ }^{1}$, J I Paley ${ }^{1}$, Y Peysson ${ }^{2}$ and the TCV Team ${ }^{1}$ \\ ${ }^{1}$ Ecole Polytechnique Fédérale de Lausanne (EPFL), Centre de Recherches en Physique des \\ Plasmas, Association EURATOM - Confédération Suisse, CH - 1015 Lausanne, Switzerland \\ ${ }^{2}$ CEA, IRFM, F-13108 Saint-Paul-lez-Durance, France \\ E-mail: loic.curchod@epfl.ch
}

Received 22 February 2011, in final form 2 August 2011

Published 6 October 2011

Online at stacks.iop.org/PPCF/53/115005

\begin{abstract}
Modulation of the amplitude of externally injected electron cyclotron (EC) power is a frequent method used to determine the radial power deposition profile in fusion plasmas. There are many tools to analyze the plasma response to the power modulations under quasi-stationary conditions. This paper focuses on the unique ability of the break-in-slope (BIS) method to retrieve a quasiinstantaneous estimate of the power deposition profile at each power step in the modulation, an outcome particularly relevant to track the power deposition location under non-stationary conditions. Here, the BIS analysis method is applied to the signals of a fast and high radial resolution wire-chamber soft $\mathrm{x}$-ray camera in the Tokamak à Configuration Variable (TCV) where the plasma magnetic configuration and thus the $\mathrm{EC}$ resonance location are varied during the plasma discharge. As a step to validate this technique before real-time control experiments, the time-varying EC power deposition location of a single beam is successfully monitored by off-line BIS analysis. Simultaneous tracking of deposition locations of two EC beams gives promising results.
\end{abstract}

(Some figures in this article are in colour only in the electronic version)

\section{Introduction}

The assessment of the radio frequency (RF) power deposition profile from experimental data is of primary importance for the interpretation of plasma auxiliary heating results and for the comparison of experiments with simulations. In particular, local temperature and current profile tailoring with electron cyclotron resonant heating $(\mathrm{ECH})$ and current drive (ECCD) allows the control of the sawtooth instability $[1,2]$ and the suppression of neo-classical tearing modes (NTMs) in tokamak plasmas [3,4]. The understanding of the involved stabilization 
mechanism requires a precise knowledge of the power deposition location (either absolute or relative to the mode location). A widespread method to retrieve the absorbed power profile is to analyze the plasma response to modulation of the injected power amplitude. Many signal analysis methods have led to conclusive results in previous heating and heat transport studies under stationary conditions: Fourier analysis $[5,6]$ or harmonic response identification methods (HRIM) [7] of the plasma response (with or without suppression of the sawtooth perturbation using singular value decomposition and system identification, SI-SVD), as well as cross-correlation or cross-power spectral density (CPSD) of the plasma response with the modulation reference waveform [8-10]. Under non-stationary conditions, a high time resolution estimate of the EC beam(s) absorption location(s) is necessary, for example in cases where the microwave injection angles are controlled by a real-time feedback loop [11]. But Fourier and correlation methods need a finite number of power modulation cycles to yield a significant result. Conversely, the break-in-slope (BIS) method is based on the estimation of the instantaneous change in the time derivative of the plasma kinetic energy at the power switch-on/off times [12-14], thus providing an estimated power deposition profile for each power step in the modulation.

Moreover, natural plasma oscillations like the sawtooth activity can heavily hamper the use of Fourier analysis methods, especially when frequency locking occurs between the power modulation and the sawtooth. In such cases, the BIS analysis allows us to select individual power modulations avoiding the time of the sawtooth crash and the related heat pulse propagation.

Under stationary conditions, experimental EC power deposition profiles measured in the Wendelstein 7-A stellarator using the BIS analysis of the temperature modulation have been successfully compared with the power deposition profiles from a ray-tracing code [15]. The present paper focuses on the ability of the BIS method to retrieve the experimental RF power deposition profile of X2 modulated ECH (MECH) under non-stationary conditions, using each power step. The BIS method is applied to the soft x-ray profiles from a fast and high radial resolution wire-chamber camera. Since soft x-ray signals are used (no electron temperature diagnostics with comparable temporal and spatial resolutions is available at TCV), the power deposition location (defined as the location of the power deposition profile maximum) rather than the full power deposition profile information is retrieved here. The BIS method results are compared with the results of the HRIM analysis of the same soft x-ray profiles and of numerical simulations.

In the following sections, it is shown under which conditions the simplest form of the BIS analysis can be applied and the HRIM analysis is presented. The experimental setup is described in section 3. In section 4, off-line BIS analysis is applied to the signals from a fast and high radial resolution soft $\mathrm{x}$-ray camera to monitor the power deposition locations of one and then two simultaneous EC power beams injected in plasmas moving in front of the launching antennas. The experimental results are compared with ray-tracing simulations. A discussion of the results and the conclusions are given in the last two sections.

\section{Analysis methods}

\subsection{The break-in-slope (BIS) analysis}

The BIS analysis to determine the auxiliary power absorbed by a given species $\alpha$ is based on the energy density conservation equation for that particular species:

$$
\frac{\partial \varepsilon_{\alpha}}{\partial t}+\nabla \cdot\left(\varepsilon_{\alpha} \boldsymbol{u}_{\alpha}\right)=Q_{\alpha}-\mathbb{P}_{\alpha}: \nabla \boldsymbol{u}_{\alpha}-\nabla \cdot \boldsymbol{q}_{\alpha}
$$


with

$$
\varepsilon_{\alpha}(\rho, t) \equiv \frac{3}{2} n_{\alpha}(\rho, t) T_{\alpha}(\rho, t)
$$

the local kinetic energy density, $\boldsymbol{u}_{\alpha}$ the fluid velocity, $Q_{\alpha}$ the power density term accounting for the particles' energy equipartition as well as for the power sources (like the external heating $P_{\text {ext }}=P_{\mathrm{aux}}+P_{\mathrm{OH}}+\cdots$ ) and sinks (like the radiation losses $P_{\mathrm{rad}}$ ), $\mathbb{P}_{\alpha}$ the pressure tensor, $\boldsymbol{q}_{\alpha}$ the heat flux density and $\nabla \equiv \partial / \partial \boldsymbol{x}$ the spatial partial derivative operator. All quantities in equation (1) are functions of the radial coordinate $\rho$ (i.e. $P_{\text {aux }}(\rho, t)$, etc). Assuming that the convective, diffusive and viscous terms as well as the equipartition power, the losses and the Ohmic source all have a typical evolution time longer than one period of the auxiliary power modulation, the energy density conservation equation (1) can be simplified to [13]

$$
\frac{\partial \varepsilon_{\alpha}}{\partial t} \simeq P_{\mathrm{aux}}
$$

If in addition the density profile remains constant mainly around the power step happening at time $t_{0}$ (except for longer time scales due to the electron density pump-out by the ECH), i.e. $n_{\alpha}(\rho, t)=n_{\alpha}\left(\rho, t_{0}\right) \equiv n_{\alpha 0}(\rho)$, equation (3) can be further simplified to

$$
\frac{3}{2} n_{\alpha 0} \frac{\partial T_{\alpha}}{\partial t} \simeq P_{\text {aux }}
$$

yielding a linear time evolution for $T_{\alpha}(\rho, t)$. In other words, the simplest form of the BIS analysis assumes that, if the heating power modulation is fast enough, the plasma temperature profile has a prompt linear response to the breaks in the auxiliary heating power amplitude. Linear fits can then be performed on the $T_{\alpha}(\rho, t)$ time traces before and after the power step $\Delta P_{\text {aux }}(\rho)$ at time $t_{0}$ :

$$
\begin{cases}T_{\alpha, 1}=a_{1}\left(t-t_{0}\right)+b_{1} & \text { for } t<t_{0}, P_{\mathrm{aux}, 1}, \\ T_{\alpha, 2}=a_{2}\left(t-t_{0}\right)+b_{2} & \text { for } t>t_{0}, P_{\mathrm{aux}, 2}=P_{\mathrm{aux}, 1}+\Delta P_{\mathrm{aux}},\end{cases}
$$

where $a_{1,2}$ and $b_{1,2}$ are functions of $\rho$. According to (4), the variation $\Delta P_{\text {aux }}(\rho)$ in the locally absorbed power is then proportional to the jump in the slope of the fits at time $t_{0}$ :

$$
\Delta P_{\mathrm{aux}} \simeq \frac{3}{2} n_{\alpha 0} \Delta\left(\frac{\partial T_{\alpha}}{\partial t}\right)_{t_{0}} \simeq \frac{3}{2} n_{\alpha 0}\left(a_{2}-a_{1}\right) .
$$

Applied to the signals of a temperature diagnostic with multiple channels or lines-of-sight viewing at different flux surfaces, the BIS analysis thus provides an estimated heating profile for each power step, i.e. at a frequency of the order of the power modulation frequency (typically a few $100 \mathrm{~Hz}$ ), which is particularly well suited for fast time-varying scenarios. Since it assumes that the break in the slope of the plasma response is simultaneous with the power steps, the simple BIS method reveals the direct heating location of the species of interest (e.g. electrons in the case of ECH). Any temperature response not in phase with the power modulation (e.g. collisional heating) is averaged-out.

The BIS analysis can be improved to retrieve and take into account the delay of the break in the slope with respect to the power step time (BIS* method) [14]. This BIS* profile then reveals both the direct heating and the indirect heating of the species of interest, similarly to a Fourier amplitude profile.

In the case of slow power modulation or a single power step, an effective loss term accounting for the energy sources and losses (diffusion, radiation, equipartition) can be retained in equation (3) to treat the saturation in the plasma response. The simplified energy density conservation equation becomes

$$
\frac{\partial \varepsilon_{\alpha}}{\partial t}=P_{\mathrm{aux}}-\frac{\varepsilon_{\alpha}}{\tau_{\alpha}}
$$


Around the power step time $t_{0}$, the solution to this new equation is an exponential:

$$
\varepsilon_{\alpha}(\rho, t)=\varepsilon_{\alpha 0} \exp \left(-\frac{t-t_{0}}{\tau_{\alpha}}\right)+P_{\mathrm{aux}} \tau_{\alpha}\left[1-\exp \left(-\frac{t-t_{0}}{\tau_{\alpha}}\right)\right],
$$

where $\varepsilon_{\alpha 0}(\rho)=\varepsilon_{\alpha}\left(\rho, t_{0}\right)$ is the kinetic energy density profile at time $t_{0}$ and $P_{\text {aux }}(\rho)$ and $\tau_{\alpha}(\rho)$ are the parameters to be determined from the exponential fits performed on the data before and after the auxiliary power jump at time $t_{0}$. The change in the absorbed auxiliary power is estimated by the slope jump of the exponential fits at time $t_{0}$. A further more general solution is proposed in [16], accounting for the heat diffusion and the variation of the heat sinks.

\subsection{The harmonic response identification method (HRIM)}

In MECH experiments, the injected RF power $P_{\mathrm{RF}}(t)$ is composed of a sinusoidally modulated part $\tilde{P}_{\mathrm{RF}}(t)$ at frequency $f_{\mathrm{mod}}=\omega / 2 \pi$, superimposed on a stationary part $\left\langle P_{\mathrm{RF}}\right\rangle_{t}$ :

$$
P_{\mathrm{RF}}(t)=\left\langle P_{\mathrm{RF}}\right\rangle_{t}+\tilde{P}_{\mathrm{RF}}(t) \quad \text { with } \tilde{P}_{\mathrm{RF}}(t)=\hat{P}_{\mathrm{RF}} \mathrm{e}^{\mathrm{i} \omega t},
$$

where $\hat{P}_{\mathrm{RF}}$ is a complex amplitude carrying information of both phase and amplitude:

$$
\hat{P}_{\mathrm{RF}}=\left|\hat{P}_{\mathrm{RF}}\right| \mathrm{e}^{\mathrm{i} \phi_{\mathrm{RF}}}
$$

Of course, the RF power excitation is generally not a purely monochromatic sinusoid but rather a sum of $N$ sinusoids at different frequencies

$$
\tilde{P}_{\mathrm{RF}}(t)=\sum_{n=1}^{N} \hat{P}_{n} \mathrm{e}^{\mathrm{i} \omega_{n} t}
$$

where $\hat{P}_{n}$ is the complex amplitude at the angular frequency $\omega_{n}$. For example, in the MECH experiments presented in this work, the EC power is modulated with a square waveform with fundamental angular frequency $\omega$ and a 50\% duty cycle. In this case, mainly the odd harmonic numbers $\omega_{n}=n \omega$ with $n=1,3,5, \ldots$ are excited.

The MECH induces oscillations of the electron temperature at the same angular frequency $\omega$ as the excitation (and possibly at other frequencies if the system is non-linear) such that the electron temperature evolution also splits into stationary and modulated parts:

$$
T_{\mathrm{e}}(t)=\left\langle T_{\mathrm{e}}\right\rangle_{t}+\tilde{T}_{\mathrm{e}}(t) \quad \text { with } \tilde{T}_{\mathrm{e}}(t)=\hat{T}_{\mathrm{e}} \mathrm{e}^{\mathrm{i} \omega t} .
$$

The complex amplitude $\hat{S}(\omega)$ of any experimental signal $S(t)$ can be estimated by the standard Fourier transform:

$$
\hat{S}(\omega)=\int_{-\infty}^{+\infty} S(t) \mathrm{e}^{\mathrm{i} \omega t} \mathrm{~d} t .
$$

But in practical MECH experiments, the number of modulation cycles available for the oscillation analysis is finite and not necessarily an integer, which will cause truncation problems in a standard Fourier analysis. Moreover, the fast modulation of the plasma response induced by the EC power modulation is often superimposed on a slow drift of the signal, which will strongly bias the Fourier components of the signal, in particular if the number of modulation cycles is small. A HRIM accounting for these effects is presented in appendix A.1 of [7] and in appendix 3 of [17]. It consists in expressing the signal $S(t)$ in the form

$S^{\prime}\left(t^{\prime}\right)=\mathcal{R}\left[\langle\hat{S}\rangle_{t}\right] \cos \left(\omega t^{\prime}\right)-\mathcal{I}\left[\langle\hat{S}\rangle_{t}\right] \sin \left(\omega t^{\prime}\right)+\langle S\rangle_{t}+\left\langle\frac{\mathrm{d} S}{\mathrm{~d} t}\right\rangle_{t}\left(t-t^{\prime}\right)$

for a time interval $t^{\prime}=[t \pm(\Delta T / 2)]$ around the time $t$, where the real and imaginary parts $\mathcal{R}\left[\langle\hat{S}\rangle_{t}\right]$ and $\mathcal{I}\left[\langle\hat{S}\rangle_{t}\right]$ of the time average complex amplitude $\hat{S}(\omega)$, as well as the time averages 
$\langle S\rangle_{t}$ and $\langle(\mathrm{d} S / \mathrm{d} t)\rangle_{t}$ of the signal $S(t)$ and of its time derivative are assumed to remain constant in the time interval considered. The parameters $\mathcal{R}\left[\langle\hat{S}\rangle_{t}\right], \mathcal{I}\left[\langle\hat{S}\rangle_{t}\right],\langle S\rangle_{t}$ and $\langle(\mathrm{d} S / \mathrm{d} t)\rangle_{t}$ are adjusted by minimizing the cost function

$$
\frac{1}{\Delta T} \int_{1-\frac{\Delta T}{2}}^{1+\frac{\Delta T}{2}}\left(S\left(t^{\prime}\right)-S^{\prime}\left(t^{\prime}\right)\right)^{2} \mathrm{~d} t^{\prime}
$$

After time discretization, the minimization procedure is expressed in matrix form and yields an estimate of the signal complex amplitude at the angular frequency $\omega$ without truncation and drift errors. For an infinite time interval (i.e. $\Delta T \rightarrow \infty$ ), the solution tends to the Fourier coefficients. If the experimental conditions are not stationary and the parameters in equation (14) are expected to vary, the total time interval during which $\mathrm{MECH}$ is applied can be divided into $M$ time intervals $t^{\prime}=\left[t_{m} \pm(\Delta T / 2)\right]$ centered on $t_{m}$ with $m=1, \ldots, M$ and the complex amplitude $\hat{S}(\omega)$ is evaluated for each $t_{m}$. The time interval division can be performed with or without overlapping, i.e. for $\Delta T>t_{m}-t_{m+1}$ or for $\Delta T \leqslant t_{m}-t_{m+1}$, respectively.

Finally, if the RF power modulation waveform is a combination of several harmonics of the fundamental excitation frequency (e.g. a square modulation), the HRIM analysis (14) can be performed for each harmonic frequency independently, or for all harmonics at once (both methods yielding essentially the same result):

$S^{\prime}\left(t^{\prime}\right)=\sum_{n=1}^{N} \mathcal{R}\left[\left\langle\hat{S}_{n}\right\rangle_{t}\right] \cos \left(n \omega t^{\prime}\right)-\mathcal{I}\left[\left\langle\hat{S}_{n}\right\rangle_{t}\right] \sin \left(n \omega t^{\prime}\right)+\langle S\rangle_{t}+\left\langle\frac{\mathrm{d} S}{\mathrm{~d} t}\right\rangle_{t}\left(t-t^{\prime}\right)$.

\section{Experimental setup}

In the Tokamak à Configuration Variable $\left(\mathrm{TCV}, R_{0}=0.88 \mathrm{~m}, a \leqslant 0.255 \mathrm{~m}, B_{\varphi} \leqslant 1.54 \mathrm{~T}\right.$, $I_{\varphi} \leqslant 1.1 \mathrm{MA}$, edge plasma elongation $0.9 \leqslant \kappa_{a} \leqslant 2.8$, edge plasma triangularity $-0.8 \leqslant$ $\delta_{a} \leqslant 0.9$ ), the toroidal magnetic field value, the position of the plasma column as well as the shape of its cross-section can be programmed to change during a plasma discharge. The flexibility of the plasma shaping is provided by 16 independent poloidal field coils [18].

The TCV second harmonic EC power system (at $f=82.7 \mathrm{GHz}$ ) is equipped with two clusters of three gyrotrons of 0.5 MW nominal power each [19]. The power amplitude of both clusters can be modulated independently. The power in extraordinary $(\mathrm{X})$ mode polarization is then injected into the plasma via six transmitting antenna referred to as launchers. Each launcher can be steered independently with two degrees of freedom (one of which can be varied during the plasma discharge), providing a full coverage of the plasma cross-section for on-axis and off-axis ECH for all plasma positions, as well as co-/counter-ECCD. Four launchers are located at the upper lateral side of the TCV vacuum vessel (with their longitudinal axis at $Z_{\text {launcher }}=45.5 \mathrm{~cm}$ ) and two launchers are located at the equatorial side (with their longitudinal axis at $Z_{\text {launcher }}=0.0 \mathrm{~cm}$ ), as indicated in figure $1(a)$. In addition, up to $1.5 \mathrm{MW}$ of third harmonic EC power in X-mode (X3) can be launched along the EC resonance via a steerable mirror installed on top of the TCV vacuum vessel. The flexibility of both TCV plasma magnetic configuration and EC power system allows the variation of the normalized radius at which the EC wave-particle resonance takes place.

The electron density and temperature profiles are measured by the Thomson scattering (TS) diagnostic [20] every $25 \mathrm{~ms}$, the density being absolutely calibrated against a far infrared (FIR) interferometer [21]. The plasma magnetic equilibria are reconstructed by the LIUQE code $[22,23]$ using TCV magnetic measurements, at the same time points as the TS measurements.

In the following sections, we study the ability of the BIS analysis to retrieve the location of the maximum power deposition at a high time rate and thus to track time-varying deposition 

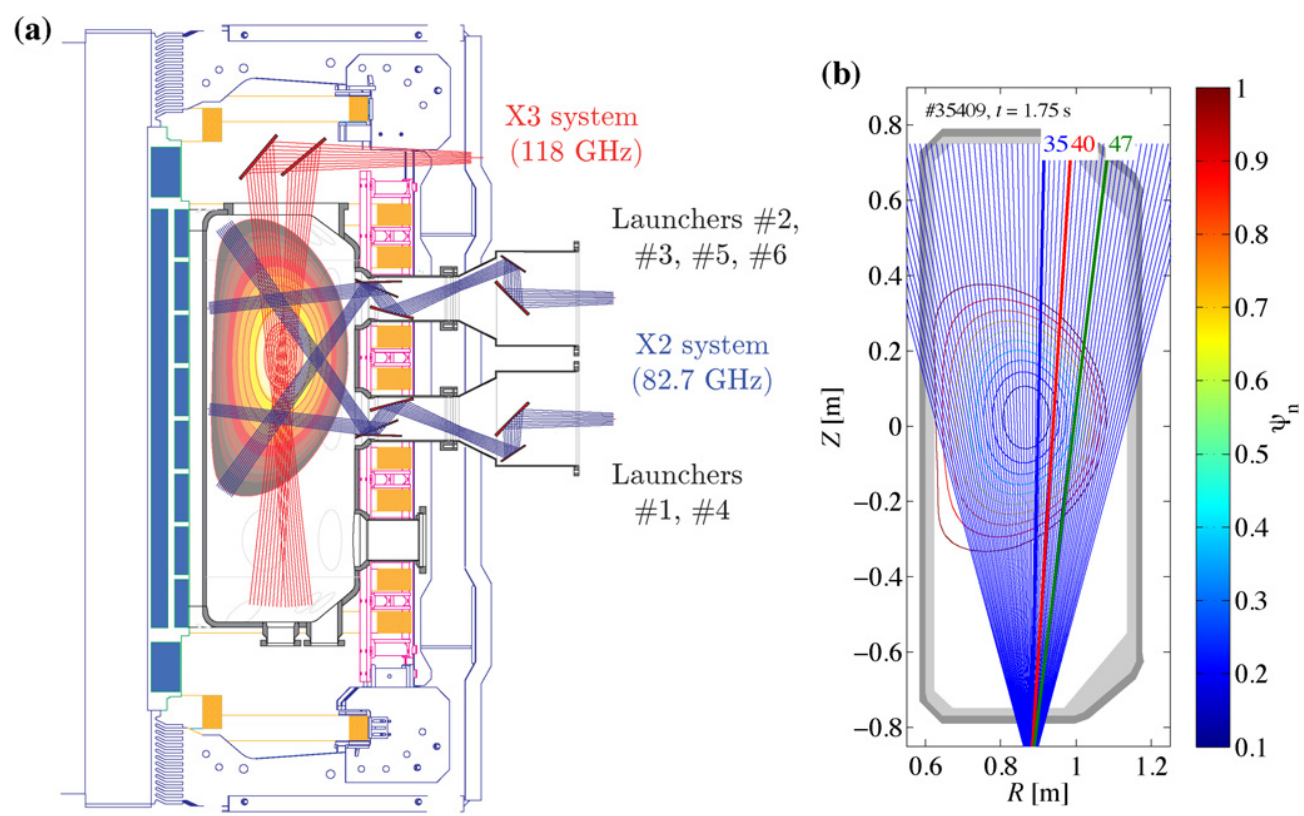

Figure 1. TCV EC system and high-resolution soft x-ray diagnostic. (a) Poloidal view of TCV vacuum vessel (in gray) showing the equatorial and upper lateral launchers of the TCV second harmonic EC power system, as well as the top launcher for the third harmonic EC power. Superimposed on the flux surface contours of a limited plasma (in red), the blue and red rays indicate the range of poloidal beam trajectories (in vacuum) that can be achieved thanks to the steerable mirrors of the X2 and X3 launchers, respectively. Since the X2 launchers can rotate on their horizontal axis, the equatorial (respectively upper lateral) launchers can also inject EC power with a downward (respectively upward) direction. (b) The 64 lines-of-sight of the multiwire proportional soft $\mathrm{x}$-ray detector (DMPX) superimposed on the normalized poloidal flux contours of TCV plasma analyzed in section 4.1. Lines-of-sight \#35, 40 and 47 are highlighted in blue, red and green, respectively. TCV shot \#35409.

locations. This is tested in MECH scenarios in TCV with one and then two simultaneous X2 power beams injected at fixed angles in plasmas programmed to sweep vertically in front of the launching antennas.

The BIS analysis is applied to the traces of a multiwire proportional soft $\mathrm{x}$-ray detector (DMPX) located under the TCV vacuum vessel and having both high temporal and radial resolutions $(200 \mathrm{kHz}$ acquisition frequency, 64 lines-of-sight with $7.9 \mathrm{~mm}$ average spacing at TCV midplane) [24], as indicated in figure $1(b)$. As shown in figure $2(b)$, the DMPX detector has a detection probability $p(v) \geqslant 5 \%$ for photons in the range $h v=1.5-30 \mathrm{keV}$ with a major peak of sensitivity in the range $h v=1.5-6 \mathrm{keV}$, where $v$ is the emitted frequency and $h$ is the Planck constant. Plasma radiation emission in the soft $\mathrm{x}$-ray range ( $h v=0.12-12 \mathrm{keV}$ ) originates from three processes: bremsstrahlung (free-free electron-ion Coulomb collisions), recombination (free-bound electron-ion collisions) and line radiation (bound-bound transition of an electron from an excited state to a less energetic state). The bremsstrahlung and recombination radiation spectra are continuous in frequency. In the TCV plasmas studied here, the central electron temperature is typically $T_{\mathrm{e} 0} \geqslant 1 \mathrm{keV}$, as indicated in figures 3 and 8 , and the following condition is thus fulfilled:

$$
T_{\mathrm{e} 0} \gg Z_{\mathrm{eff}}^{2} R_{\mathrm{y}} \simeq 122 \mathrm{eV},
$$



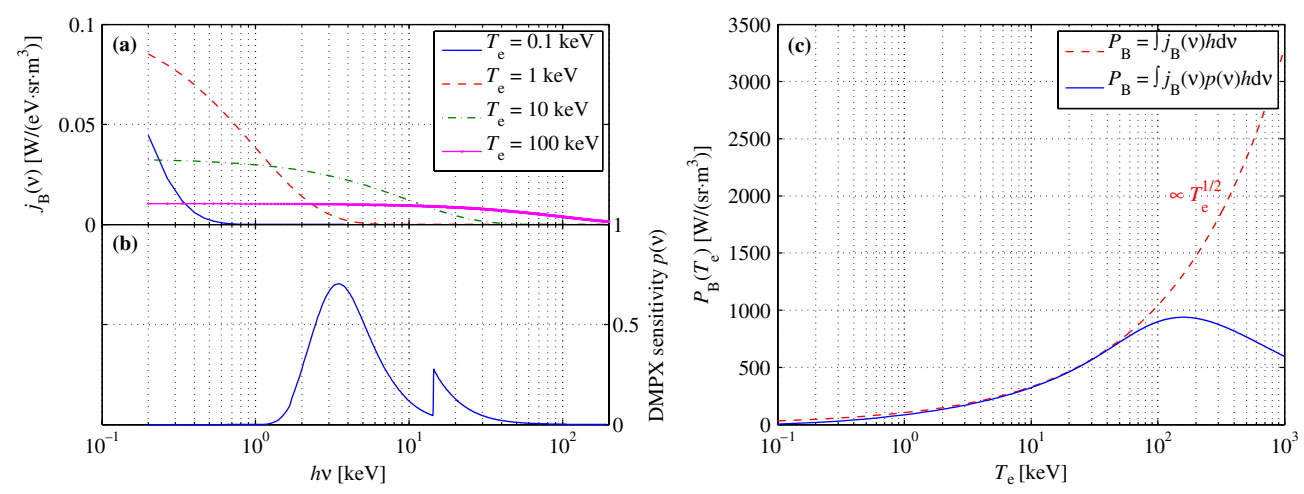

Figure 2. Soft x-ray bremsstrahlung power density. (a) Bremsstrahlung spectral emissivity $j_{\mathrm{B}}$ as a function of the photon energy $h v$ for $n_{\mathrm{e}}=3 \times 10^{19} \mathrm{~m}^{-3}, Z_{\mathrm{eff}}=3, g_{\mathrm{ff}}=1$ and several values of the electron temperature $T_{\mathrm{e}}$. (b) DMPX sensitivity $p$ (also called detection probability) as a function of the photon energy $h v$. (c) Integrated bremsstrahlung power density $P_{\mathrm{B}}$ as a function of the electron temperature $T_{\mathrm{e}}$, accounting (blue solid curve) or not accounting (red dashed curve) for the DMPX sensitivity given in $(b)$. In the first case, the power density $P_{\mathrm{B}}$ monotonically increases with $T_{\mathrm{e}}$ up to $T_{\mathrm{e}} \simeq 160 \mathrm{keV}$.

where $R_{\mathrm{y}} \simeq 13.6 \mathrm{eV}$ is the Rydberg energy and $Z_{\mathrm{eff}} \equiv \sum_{\mathrm{i}} n_{\mathrm{i}} Z_{\mathrm{i}}^{2} / n_{\mathrm{e}} \simeq 3$ is the plasma effective charge (the quasi-neutrality condition $n_{\mathrm{e}}=\sum_{\mathrm{i}} n_{\mathrm{i}} Z_{\mathrm{i}}$ is invoked here). As a consequence, the recombination radiation is negligible for all frequencies $v$ compared with the bremsstrahlung radiation [25]. Moreover, the line radiation from light impurities (carbon and oxygen) is important only in the ultra-soft x-ray spectral range $(h v \leqslant 1 \mathrm{keV})$. The DMPX diagnostic thus measures mainly bremsstrahlung radiation.

Assuming a Maxwellian electron velocity distribution, the bremsstrahlung spectral emissivity $j_{\mathrm{B}}(v)$ (power per unit solid angle per unit volume per unit energy of the emitted photons) can be written as [25]

$$
j_{\mathrm{B}}(\nu)=g_{\text {ff }} n_{\mathrm{e}}^{2} Z_{\mathrm{eff}} \frac{e}{h}\left(\frac{e^{2}}{4 \pi \epsilon_{0}}\right)^{3} \frac{8 \pi}{3 \sqrt{3} m_{\mathrm{e}}^{2} c^{3}}\left(\frac{2 m_{\mathrm{e}}}{\pi e T_{\mathrm{e}}}\right)^{1 / 2} \mathrm{e}^{-\frac{h v}{T_{\mathrm{e}}}}
$$

which evaluates to

$$
j_{\mathrm{B}}(\nu)=1.22 \times 10^{-39} g_{\mathrm{ff}} n_{\mathrm{e}}^{2} Z_{\mathrm{eff}} \frac{1}{\sqrt{T_{\mathrm{e}}}} \mathrm{e}^{-\frac{h \nu}{T_{\mathrm{e}}}}\left(\mathrm{W} \mathrm{sr}^{-1} \mathrm{~m}^{-3} \mathrm{eV}^{-1}\right),
$$

where the electron density $n_{\mathrm{e}}$ is expressed in $\left(\mathrm{m}^{-3}\right)$, and the electron temperature $T_{\mathrm{e}}$ and photon energy $h v$ are expressed in $(\mathrm{eV})$. The temperature and frequency dependence of the Maxwell-averaged free-free Gaunt factor $g_{\mathrm{ff}}$ is weak [25] and $g_{\mathrm{ff}}$ is assumed to be a constant. The spectral emissivity $j_{\mathrm{B}}$ is plotted in figure $2(a)$ for $n_{\mathrm{e}}=3 \times 10^{19} \mathrm{~m}^{-3}, Z_{\text {eff }}=3, g_{\text {ff }}=1$ and several values of $T_{\mathrm{e}}$. After integration on the whole energy spectrum, the bremsstrahlung power density $P_{\mathrm{B}}$ emitted per unit solid angle and unit volume depends on the electron density and temperature and on the plasma effective charge as

$$
P_{\mathrm{B}} \equiv \int_{0}^{\infty} j_{\mathrm{B}}(v) h \mathrm{~d} v=1.22 \times 10^{-39} g_{\mathrm{ff}} n_{\mathrm{e}}^{2} Z_{\mathrm{eff}} \sqrt{T_{\mathrm{e}}}\left(\mathrm{W} \mathrm{sr}{ }^{-1} \mathrm{~m}^{-3}\right) .
$$

One needs to account for the detector sensitivity shown in figure $2(b)$ in order to evaluate the actual bremsstrahlung power density measured by the DMPX:

$$
P_{\mathrm{B}} \equiv \int_{0}^{\infty} j_{\mathrm{B}}(v) p(v) h \mathrm{~d} v
$$


Numerical integration yields the bremsstrahlung power density as a function of $T_{\mathrm{e}}$ shown in figure 2(c) (blue curve) along with the function (20) (red curve) for $n_{\mathrm{e}}=3 \times 10^{19} \mathrm{~m}^{-3}, Z_{\text {eff }}=3$, $g_{\mathrm{ff}}=1$. When both the electron density and the plasma effective charge remain constant, the bremsstrahlung power measured by the DMPX is a monotonically increasing function of the electron temperature up to $T_{\mathrm{e}} \simeq 160 \mathrm{keV}$, i.e. in the temperature range of interest. The power deposition location in MECH experiments can thus be inferred at each power step by applying the BIS method to the 64 traces of the soft x-ray power profile $I_{S X}$ measured by the DMPX diagnostic, the maximum BIS amplitude indicating the location of direct heating.

Since the DMPX is sensitive to photons with an energy $h v \geqslant 1.5 \mathrm{keV}$ and the central electron temperature in our experiments is in the range $T_{\mathrm{e}}=1-2.5 \mathrm{keV}$, the detected bremsstrahlung is generated mainly by electrons having a kinetic energy $E \geqslant T_{\mathrm{e}}$. Under our experimental conditions (low loop voltage $V_{\text {loop }} \lesssim 1 \mathrm{~V}$, medium-high central density $n_{\mathrm{e} 0}=(2-4) \times 10^{19} \mathrm{~m}^{-3}$, no EC CD), we expect these electrons to mostly belong to the thermal Maxwellian bulk, except in the power deposition region where a tail of suprathermal electrons is generated by the ECRH. Estimates using the electron distribution function calculated by the LUKE quasi-linear Fokker-Planck-equation solver code [26] in the presence of ECRH indeed show that the contribution from this suprathermal electron tail to the DMPX signal is locally significant. Yet, as ECRH is turned on, a linear growth phase for both the buildup of a suprathermal electron population and the local temperature increase (resulting from energy exchange between fast and bulk electrons) takes place during a few fast resonant electron slowing-down times, which for ECRH is longer than the thermal slowing-down time by typically one order of magnitude. It is thus not relevant for our BIS analysis whether the DMPX signal is dominated by bulk or suprathermal electron contributions, or even a mixed contribution.

Finally, the measured x-ray power $I_{\mathrm{SX}}$ is line-integrated (i.e. integrated over the solid angle and the observation volume of each of the 64 DMPX chords) and the resulting soft x-ray profile is a non-local information. However, the signal of each DMPX line-of-sight originates mainly from the plasma flux surface which is tangential to the line-of-sight and is therefore tagged with the corresponding flux surface label $\rho_{\mathrm{vol}}$. Here, the normalized minor radius $\rho_{\mathrm{vol}}$ of a flux surface is defined as

$$
\rho_{\mathrm{vol}} \equiv \sqrt{\frac{V}{V_{a}}},
$$

where $V$ is the volume of the flux surface and $V_{a}$ is the total volume of the plasma, such that $\rho_{\mathrm{vol}}=0$ on the magnetic axis and $\rho_{\mathrm{vol}}=1$ on the last closed flux surface (LCFS). Note that this approximation is valid especially in the region of significant temperature and density gradients, i.e. in the plasma periphery. In the plasma core where the profiles are flatter, the assumption that the main contribution for each chord comes from the tangential surface may fail and the error due to this approximation may become important.

\section{Ray-tracing simulations and experimental results}

\subsection{Single EC power deposition location tracking}

In this experiment, the equilibrium is a limited plasma with medium shaping $\left(\kappa_{a} \simeq 1.55\right.$, $\left.\delta_{a} \simeq 0.45\right)$ and low plasma current $I_{\mathrm{p}}=135 \mathrm{kA}$ yielding a high edge safety factor value $q_{a}>9$ and a central safety factor marginally above $q_{0}=1$. This results in a fast and small amplitude sawtooth activity. When the off-axis MECH starts at $t=0.35 \mathrm{~s}$, the sawtooth activity becomes quasi-indistinguishable on the soft x-ray traces. Hence, the sawtooth perturbation 


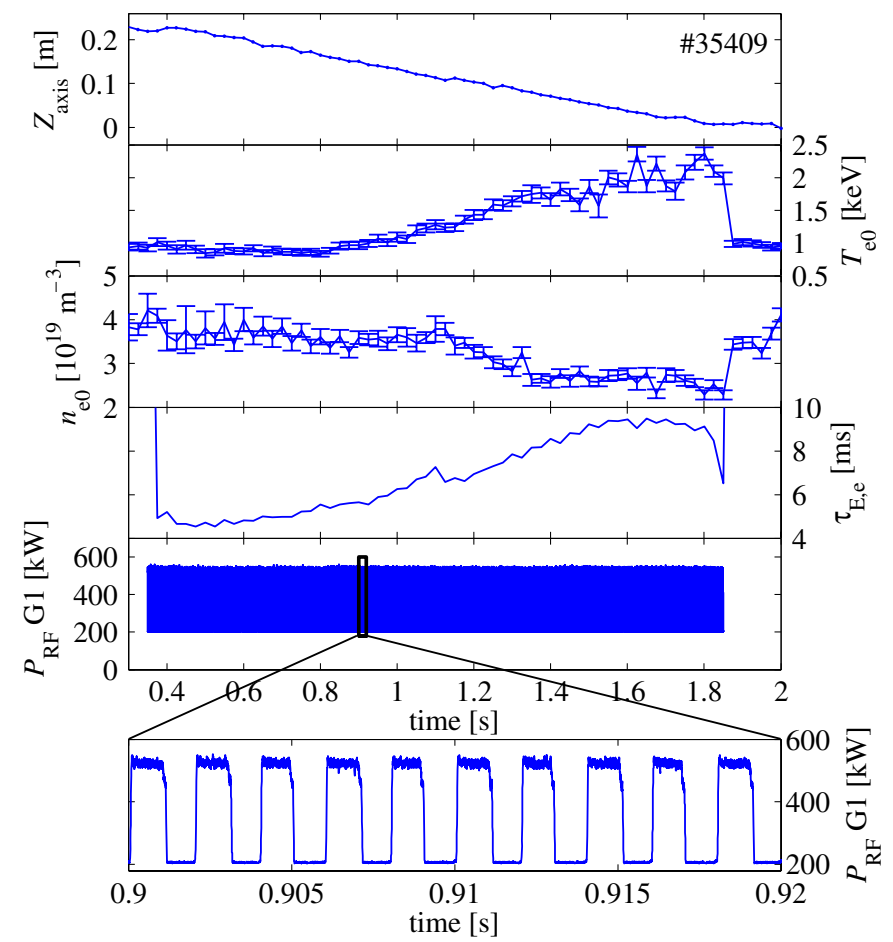

Figure 3. Plasma vertical position sweep. From top to bottom: time evolution of the magnetic axis vertical position (LIUQE reconstruction code), the central electron temperature and density (TS, corrected by FIR), the electron energy confinement time and the EC power injected from launcher 1 , modulated at $f_{\text {mod }}=500 \mathrm{~Hz}$ with a duty cycle $d_{\mathrm{c}}=50 \%$. The black rectangle defines the zoom region on the square power modulation shown in the bottom subplot. The confinement time, increasing while the deposition is getting more central, is calculated for an average ECH power $\bar{P}_{\mathrm{RF}}=365 \mathrm{~kW}$. The toroidal magnetic field and the plasma current are maintained constant at $B_{\varphi}=1.45 \mathrm{~T}$ and $I_{\mathrm{p}} \simeq 135 \mathrm{kA}$, respectively, while the plasma vertical position is swept down from $Z_{\text {axis }}=24 \mathrm{~cm}$ to $0 \mathrm{~cm}$ in the time interval $t=[0.2 ; 2.0] \mathrm{s}$. TCV shot \#35409.

on the BIS estimate is negligible. As we will see later on, this assumption is true as long as the $\mathrm{X} 2$ power is not deposited at the plasma center. The nominal value of the magnetic field is $B_{\varphi}=1.45 \mathrm{~T}$ such that the second harmonic electron cyclotron resonance (ECR) is located close to the plasma axis at $R \simeq 0.85 \mathrm{~m}$ as indicated on the poloidal views in figure 4 .

All parameters of the magnetic equilibrium are kept constant, except for the magnetic axis vertical position, which is programmed to sweep down from $Z_{\text {axis }}=24 \mathrm{~cm}$ to $Z_{\text {axis }}=0 \mathrm{~cm}$ in the time interval $t=[0.2 ; 2.0] \mathrm{s}$ in front of the equatorial launcher L1 injecting one X2 EC power beam, see figure 3 . The power amplitude is modulated at a frequency $f_{\text {mod }}=500 \mathrm{~Hz}$ with a square waveform, a depth $\Delta P_{\mathrm{RF}}=320 \mathrm{~kW}$ (between 205 and $525 \mathrm{~kW}$ ) and a duty cycle $d_{\mathrm{c}}=50 \%$. The launcher angles are kept fixed for a quasi-horizontal injection in the poloidal plane with a $9^{\circ}$ upward poloidal angle. The beam absorption location is thus expected to move from off-axis to the central normalized radius when the plasma sweeps down in front of the launcher.

Indeed, simulations from the $\mathrm{C} 3 \mathrm{PO}$ ray-tracing code [27] and the LUKE quasi-linear Fokker-Planck-equation solver code [26] for successive equilibria along the plasma discharge evolution indicate a displacement of the beam-plasma resonant interaction from $\rho_{\mathrm{vol}}=0.63$ to $\rho_{\mathrm{vol}}=0.07$, as indicated in figure 4 . All simulations were performed using the experimental 

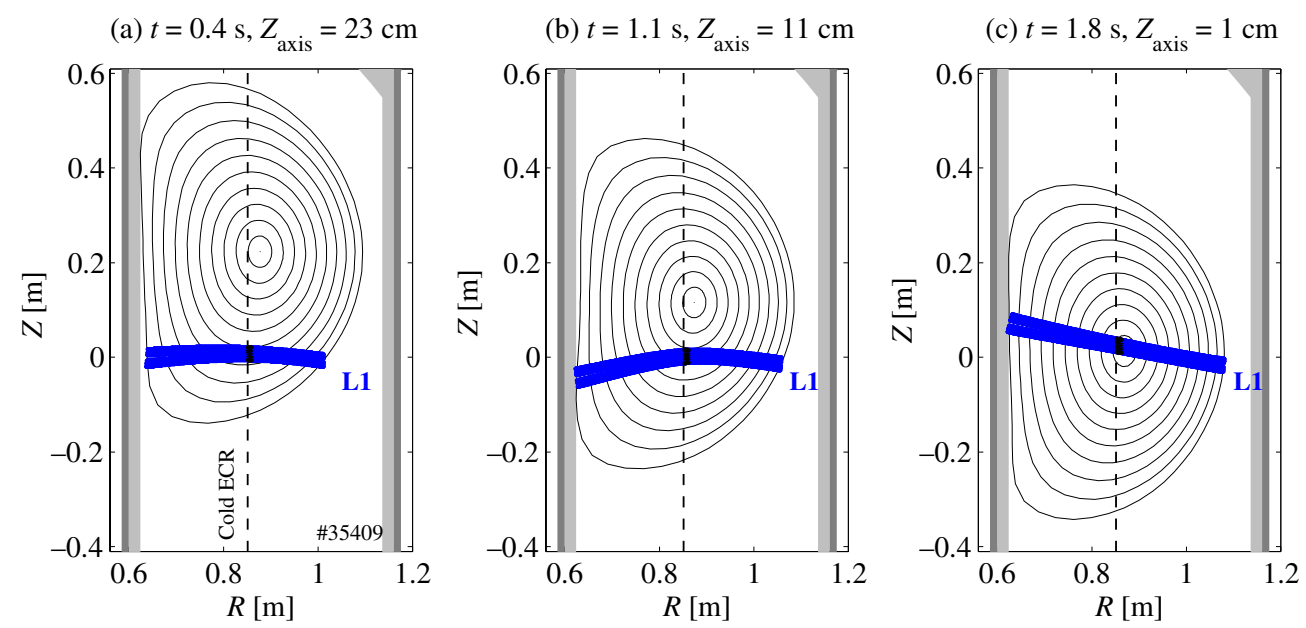

Figure 4. Time-varying deposition location. Poloidal trajectory of $\mathrm{X} 2$ ray-tracing simulations with the C3PO code [27] for a 12-ray beam injected from the equatorial launcher L1, at times (a) $t=0.4 \mathrm{~s},(b) 1.1 \mathrm{~s}$ and $(c) 1.8 \mathrm{~s}$ when the plasma magnetic axis is located at $Z_{\text {axis }}=23 \mathrm{~cm}$, $11 \mathrm{~cm}$ and $1 \mathrm{~cm}$, respectively. The vertical dashed line indicates the cold EC resonance location. The location of the beam-plasma resonant interaction (black region on the right of the resonance) moves from mid-radius to the plasma core, i.e. $\rho_{\text {vol }}=0.63,0.35$ and 0.07 , respectively. TCV shot \#35409.

equilibrium and profiles data, with $Z_{\mathrm{eff}}=3, T_{\mathrm{e}} / T_{\mathrm{i}}=6$ and $500 \mathrm{~kW}$ of injected EC power. This is coherent with the progressive increase in the measured central electron temperature, from $T_{\mathrm{e} 0} \simeq 1 \mathrm{keV}$ to $T_{\mathrm{e} 0} \simeq 2.5 \mathrm{keV}$, as indicated in figure 3 . In the meanwhile, the central electron density decreases from $n_{\mathrm{e} 0} \simeq 4 \times 10^{19} \mathrm{~m}^{-3}$ to $n_{\mathrm{e} 0} \simeq 2.5 \times 10^{19} \mathrm{~m}^{-3}$ due to the ECRH density pump-out effect.

Before the BIS analysis is applied to the soft X-ray data, each of the 64 line-integrated DMPX signals is band-pass-filtered to remove the fast MHD activity perturbations and the slow time-varying component of the signal using a first-order zero-phase Butterworth filter with cutoff frequencies $f_{\text {mod }} / 2=250 \mathrm{~Hz}$ and $f_{\text {mod }}=500 \mathrm{~Hz}$. The resulting signal is the modulated part of the soft $\mathrm{x}$-ray traces:

$$
\tilde{I}_{\mathrm{SX}}(t)=I_{\mathrm{SX}}(t)-\left\langle I_{\mathrm{SX}}\right\rangle_{t} \text {. }
$$

Since we use soft $x$-ray signals here instead of electron temperature signals, the density and effective charge profiles will distort the estimated power deposition profile. In order to alleviate this difficulty, the signal is normalized along the time dimension such that all 64 soft x-ray traces take values between 0 and 1 .

In these MECH experiments, the period of the microwave power modulation $\tau_{\text {mod }}=2 \mathrm{~ms}$ is smaller than the typical electron energy confinement time $\tau_{E, \mathrm{e}}>5 \mathrm{~ms}$ and the plasma response is nearly linear, as indicated in figure 5. Thus, the simple BIS analysis based on linear fits can be used. It is applied to all DMPX channels independently. Linear fits are performed on the DMPX traces between all EC power step-up and step-down times. The break in the linear fits' slope is then calculated at each power step-up time for each DMPX chord, providing BIS amplitude profiles at a $f_{\text {mod }}=500 \mathrm{~Hz}$ rate. In a similar way, the BIS information can be deduced at the power step-down times, thus doubling the information rate to $2 f_{\text {mod }}=1 \mathrm{kHz}$.

As previously mentioned, the soft X-ray traces are labeled with the $\rho_{\mathrm{vol}}$ coordinate of the flux surface to which the DMPX lines-of-sight are tangential. With mid-radius deposition (at 


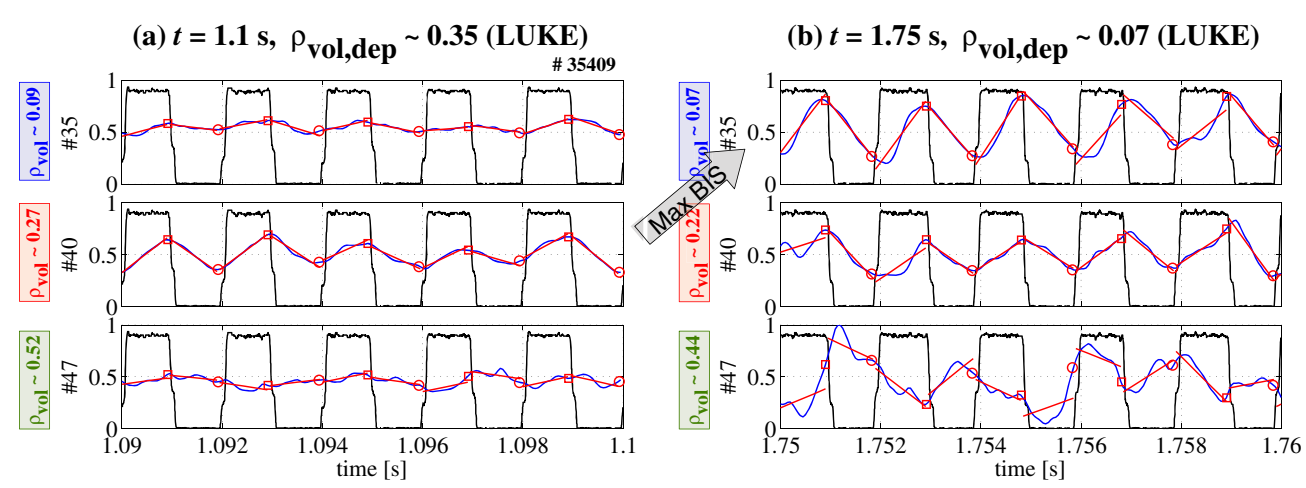

Figure 5. Displacement of the maximum slope jump amplitude. BIS analysis of the soft x-ray signal from DMPX chords \#35, 40 and 47 for $(a) t=1.1 \mathrm{~s}$ and $(b) t=1.75 \mathrm{~s}$, respectively. The chord \#35 is the most central-viewing one, as indicated in figure 1(b). The filtered and normalized soft X-ray signals (in blue) are plotted on top of the X2 square waveform (in black). Linear fits (in red) are performed between the EC power step-up and step-down times (indicated by red circles and squares). At times $t=1.1 \mathrm{~s}$ and $t=1.75 \mathrm{~s}$, the largest slope jumps take place at the mid-radiusviewing chord $\# 40\left(\rho_{\mathrm{vol}} \simeq 0.27\right)$ and at the central-viewing chord $\# 35$ ( $\left.\rho_{\mathrm{vol}} \simeq 0.07\right)$, respectively, as expected from the simulations in figure 4. TCV shot \#35409.

time $t=1.1 \mathrm{~s}$, see figure 5(a)), the largest slope jumps take place at the mid-radius-viewing chord \#40 ( $\left.\rho_{\mathrm{vol}} \simeq 0.27\right)$ as expected from the simulation in figure $4(b)$ indicating power deposition at $\rho_{\mathrm{vol}}=0.35$. With central deposition (at time $t=1.75 \mathrm{~s}$, see figure $5(b)$ ), the region of the strongest slope jumps moves to the central-viewing chord \#35 ( $\rho_{\mathrm{vol}} \simeq 0.07$ ), as expected from the simulation in figure $4(c)$ indicating power deposition at $\rho_{\mathrm{vol}}=0.07$.

Note that the plasma response to the MECH is linear close to the expected power deposition location. Far from this point, the perturbation has dissipated during the heat pulse propagation and it is not distinguishable from the background plasma fluctuations any more. This is the case for the time trace of chord $\# 47$ at $t=1.75 \mathrm{~s}$. At this radius, $\sim 40 \%$ of $\rho_{\mathrm{vol}}$ away from the power deposition location, the BIS information becomes random and is not relevant any more. One should thus use the BIS technique in the limit of a distinguishable oscillating plasma response, close to the expected power deposition location. Here, the whole soft x-ray profile is analyzed (even far from the power deposition point) for the sake of completeness and to learn about the limitation of the method.

The color map in figure 6 shows the successive BIS amplitude profiles versus time. Only the low-field side (LFS) part of the profiles is shown. In the same figure, the color contours give the local power deposition profiles calculated by the C3PO-LUKE codes. The maximum BIS amplitude location is tracked along time and filtered using a seven-pulse-cycle running median average, yielding an estimated time evolution of the power deposition location. As expected, the location of the maximum BIS amplitude clearly moves from the far off-axis to the central radius when the plasma vertical position decreases. The simulated and experimental power deposition locations are all superimposed in the last subplot of figure 6. The experimental power deposition locations $\rho_{\text {dep, ON }}$ and $\rho_{\text {dep, OFF }}$, inferred from the BIS analysis at the EC power switch-on and switch-off times, respectively, are in excellent agreement with each other, with a time average difference $\left\langle\left|\rho_{\text {dep, ON }}-\rho_{\text {dep, OFF }}\right|\right\rangle_{t} \simeq 2.3 \%$, during the whole position sweep.

The HRIM analysis is performed on the same filtered and normalized soft x-ray profiles used in the BIS analysis. The complex amplitude at $f_{\text {mod }}=500 \mathrm{~Hz}$ is calculated for each of the 64 soft $x$-ray traces in 14 successive $100 \mathrm{~ms}$ long time intervals. The HRIM analysis therefore provides one amplitude profile per $100 \mathrm{~ms}$ interval whereas the BIS analysis 


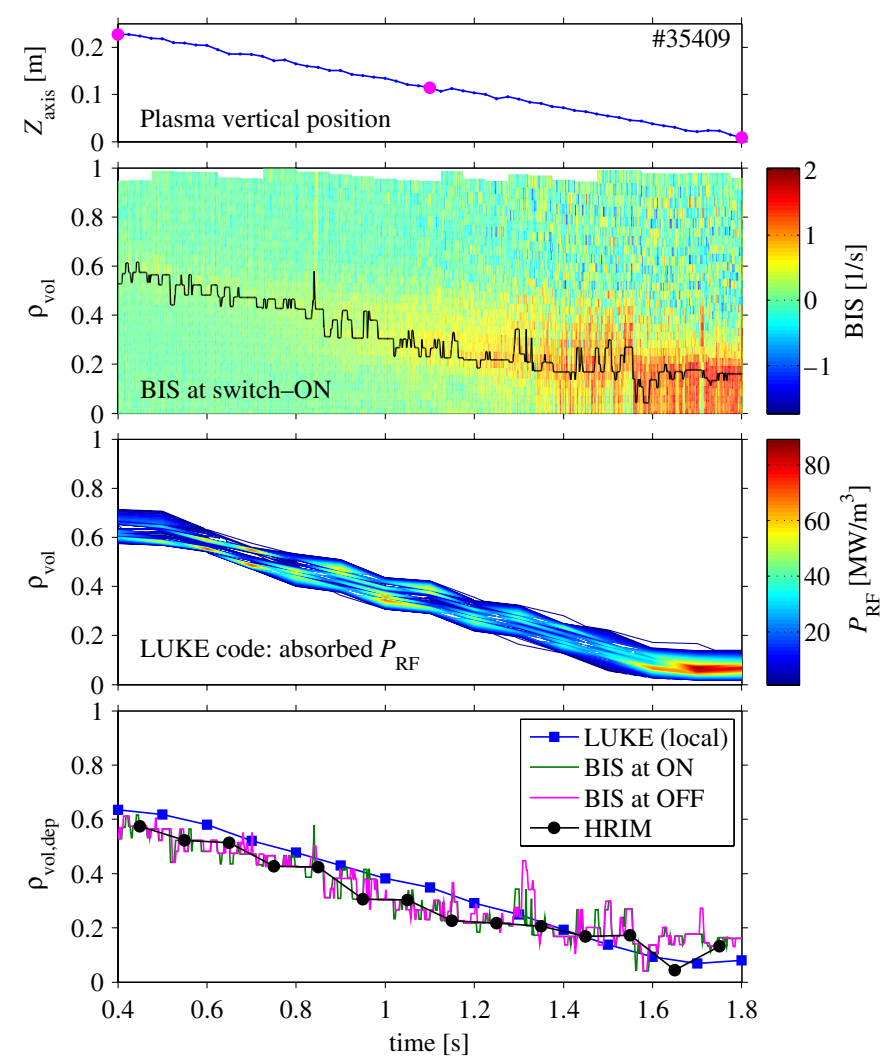

Figure 6. X2 power deposition location tracking. From top to bottom: plasma vertical position versus time (pink dots indicate the C3PO simulation times in figure 4); BIS amplitude versus time and normalized radius $\rho_{\mathrm{vol}}$ (only the LFS part of the BIS profiles is shown and the black line is a seven-pulse-cycle running median average of the maximum BIS amplitude location); local absorbed EC power density versus time and normalized radius $\rho_{\mathrm{yol}}$ from LUKE simulations; superimposed EC power deposition locations from LUKE (blue $\mathbf{\square}$ ), from BIS analysis of the soft $\mathrm{X}$-ray profiles at both EC power switch-on (green trace) and switch-off times (magenta trace), and from HRIM analysis of the soft x-ray profiles (black - ). Both BIS and HRIM analysis methods are in excellent agreement with each other and within $10 \%$ of $\rho_{\mathrm{vol}}$ with the numerical simulation. TCV shot \#35409.

yields 100 amplitude profiles in the same time (thanks to 50 power switch-on events and 50 power switch-off events). The location of the maximum soft x-ray oscillation amplitude inferred from the HRIM analysis is named $\rho_{\text {dep,HRIM }}$ and is shown in the bottom subplot of figure 6. It is in excellent agreement with the results of the BIS analysis, with a difference $\left\langle\left|\rho_{\text {dep,ON }}-\rho_{\text {dep,HRIM }}\right|\right\rangle_{t} \simeq 3.1 \%$ averaged on the whole position sweep. The soft x-ray oscillation phase inferred from the HRIM analysis (not shown here) exhibits a local minimum which also follows the power deposition location.

For off-axis deposition $\left(\rho_{\mathrm{vol}, \text { dep }} \gtrsim 0.3\right.$, i.e. $t<1.3 \mathrm{~s}$ ), the experimental results from both the BIS and the HRIM analyses are in fair agreement with the simulated power deposition location, that is $\left\langle\left|\rho_{\text {dep, ON }}-\rho_{\text {dep,LUKE }}\right|\right\rangle_{t} \simeq 8.4 \%$ more inside than the simulation result. This systematic discrepancy can be explained by the fact that the BIS and the HRIM analyses are applied to line-integrated soft X-ray data, whereas the simulated deposition profiles are local. A tomographic inversion of the soft x-ray data should cause a slight outward shift of the experimental power deposition location [28], which should improve the agreement with 


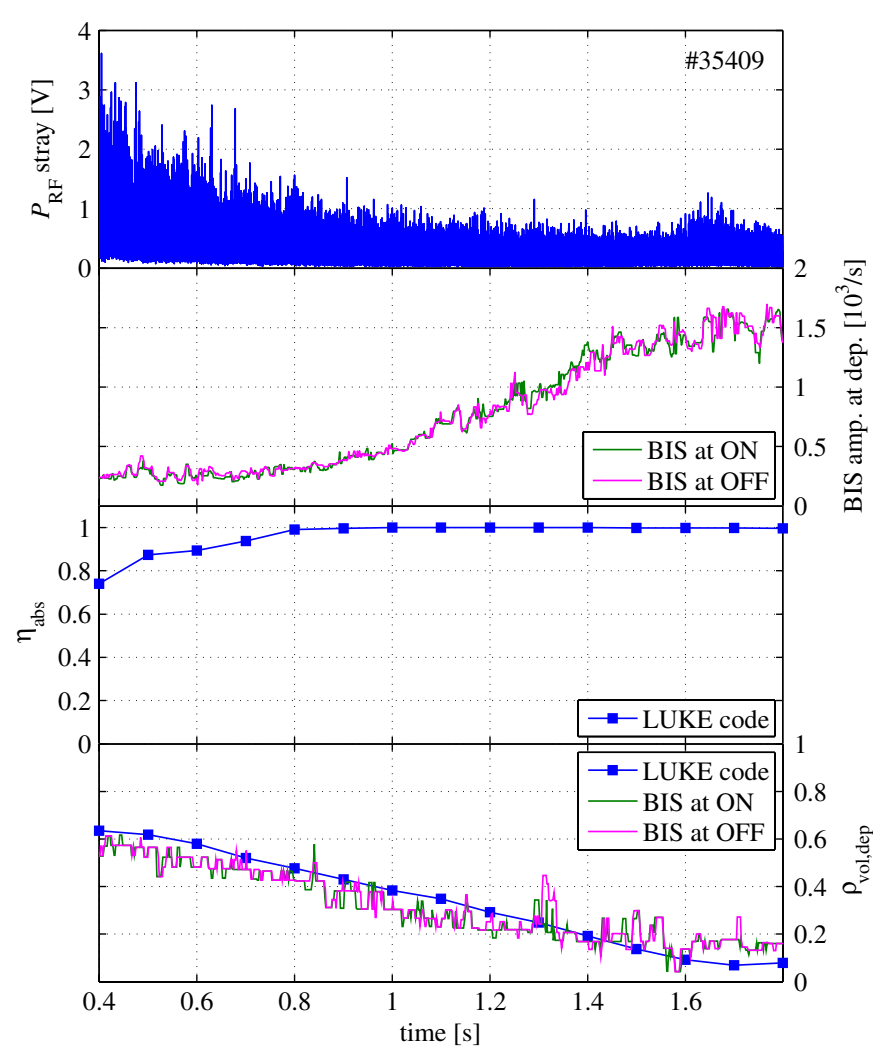

Figure 7. EC power absorption efficiency. From top to bottom: stray EC power level, maximum BIS amplitude at power switch-on and switch-off times, X2 EC power absorption efficiency calculated by the LUKE code, and power deposition location from BIS analysis and simulations. Since BIS amplitudes at switch-on and switch-off times have opposite signs, the latter is multiplied by -1 . The LUKE code indicates that the X2 power absorption increases from $74 \%$, when the beam power is deposited at the edge where the temperature is low, to $100 \%$ for central deposition. Both the level of stray EC power and the maximum BIS amplitude indicate the same trend. TCV shot \#35409.

the ray-tracing calculation. In stationary scenarios, the DMPX profile can be inverted using a Fisher-information minimization algorithm, mapping both high-field side (HFS) and LFS viewing chords on one local profile. In the present non-stationary scenarios where the flux surfaces cross different lines-of-sight as a function of time, the signal/noise ratio after inversion is poor and the modulation information is partly lost. Note that the inversion of the soft-x ray profiles is not necessary if the same diagnostic is used to determine both the location of the power deposition and the aiming point of the beam, e.g. in the case of NTM tracking and stabilization with EC power. In this case, relative position information is sufficient.

For central deposition $\left(\rho_{\mathrm{vol}, \text { dep }} \lesssim 0.3, t>1.3 \mathrm{~s}\right)$, the experimental deposition location is more outward than expected from the simulation and it undergoes larger fluctuations. In particular, the power deposition locations inferred from the BIS and the HRIM analyses do not overlap anymore. Both effects may be caused by the resurgence of the sawtooth activity or a similar periodic profile relaxation triggered by the $\mathrm{MECH}$ when it reaches the plasma center. Also, as previously mentioned in section 3, the approximation consisting in labeling the line-integrated signal with the local normalized radius $\rho_{\mathrm{vol}}$ may induce significant errors 


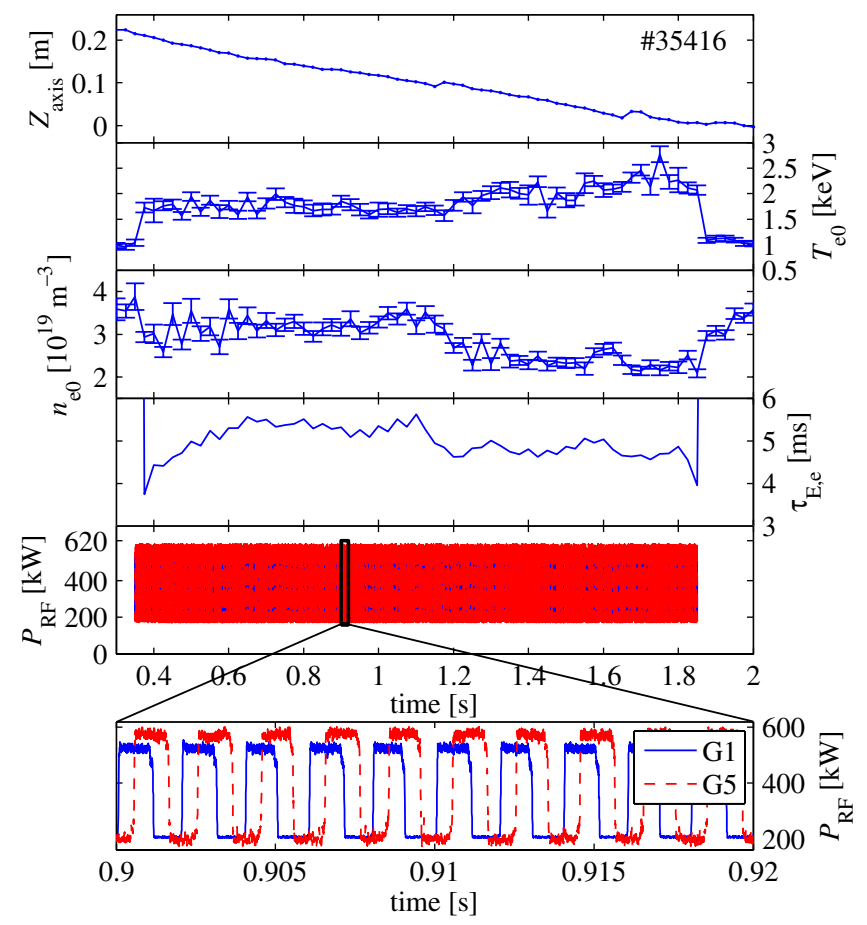

Figure 8. Plasma vertical sweep and double beam modulation. From top to bottom: same as in figure 3. In addition to the X2 EC power beam injected from the equatorial launcher L1 (blue solid $P_{\mathrm{RF}}$ trace), a X2 EC power beam is injected from the upper lateral launcher L5 (red dashed $P_{\mathrm{RF}}$ trace). Both EC power traces are square modulated at $f_{\text {mod }}=500 \mathrm{~Hz}$ with a duty cycle $d_{\mathrm{c}}=50 \%$, but with a relative phase of $\pi / 2$. The confinement time was calculated for an average ECH power $\bar{P}_{\mathrm{RF}}=750 \mathrm{~kW}$. The toroidal magnetic field and the plasma current are maintained constant at $B_{\varphi}=1.45 \mathrm{~T}$ and $I_{\mathrm{p}} \simeq 160 \mathrm{kA}$, respectively, while the plasma vertical position is swept down from $Z_{\text {axis }}=24 \mathrm{~cm}$ to $0 \mathrm{~cm}$ in the time interval $t=[0.2 ; 2.0] \mathrm{s}$. TCV shot \#35416.

in the plasma core, which can explain in part the discrepancy between the experiment and the simulation.

Although this experiment is designed rather for the fast tracking of the EC power deposition location than for a quantitative estimation of the absorption efficiency, the maximum BIS amplitude is compared qualitatively with the power absorption efficiency calculated by LUKE in figure 7. Note that the BIS amplitude at the power switch-off times is simply multiplied by -1 to compare the information of BIS amplitude at switch-on and switch-off times. At the beginning of the plasma vertical sweep, the X2 beam power is deposited at the edge where the electron temperature is low and the EC power is not fully absorbed (74\% absorption according to LUKE), as confirmed by the high level of stray EC power which provides a qualitative indication of the non-absorbed power fraction. At the end of the plasma position sweep, the LUKE code indicates that central deposition and full absorption are reached. The maximum BIS amplitude follows the same trend, increasing with more central deposition radius $\rho_{\mathrm{vol}, \mathrm{dep}}$.

\subsection{Double EC power deposition locations tracking}

The natural next step toward real-time applications of the BIS analysis method is to test the potential of simultaneous tracking of multiple power deposition locations. For this, a similar 

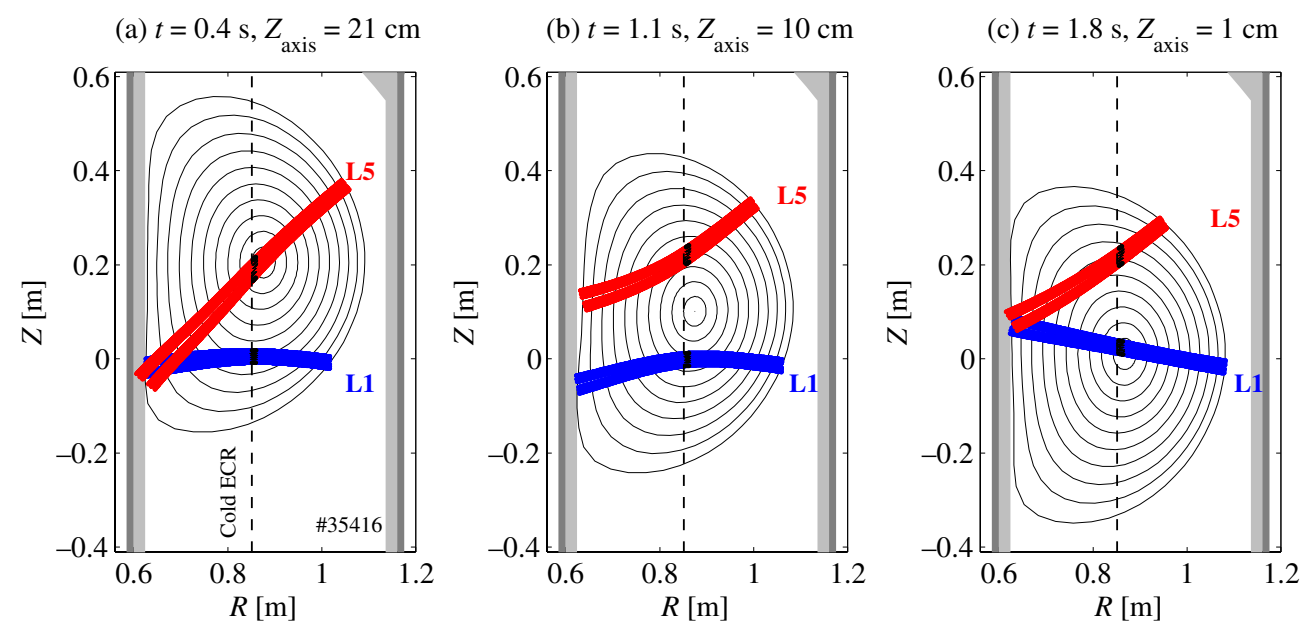

Figure 9. Double time-varying deposition locations. Poloidal trajectory of X2 ray-tracing simulations with the C3PO code [27] for 12-ray beams injected from the equatorial launcher L1 (blue) and from the upper lateral launcher L5 (red), at times $(a) t=0.4 \mathrm{~s},(b) 1.1 \mathrm{~s}$ and $(c)$ $1.8 \mathrm{~s}$ when the plasma magnetic axis is located at $Z_{\text {axis }}=21 \mathrm{~cm}, 10 \mathrm{~cm}$ and $1 \mathrm{~cm}$, respectively. The vertical dashed line indicates the cold EC resonance location. The beam-plasma resonant interaction location (black regions on the right of the resonance) moves from mid-radius to the plasma core for the beam coming from L1 and vice versa for the beam coming from L5. TCV shot \#35416.

plasma target is repeated where, in addition to the EC power beam from the equatorial launcher L1, a second EC power beam is injected into the poloidal plane from the upper lateral launcher L5 with a $30^{\circ}$ downward poloidal angle. When the plasma vertical position is swept down, the power deposition location of the first beam is expected to move from mid-radius to the center of the plasma and vice versa for the second beam, as shown by $\mathrm{C} 3 \mathrm{PO}$ ray-tracing simulations in figure 9.

In order to perform independent but simultaneous BIS analysis of the beam absorption locations, both power amplitudes are modulated with the same $500 \mathrm{~Hz}$ square waveform as in the previous experiment, but with a phase difference of $\pi / 2$, as indicated in figure 8 . The difference in the modulation depths $-\Delta P_{\mathrm{RF}}=320 \mathrm{~kW}$ for L1 and $\Delta P_{\mathrm{RF}}=375 \mathrm{~kW}$ for L5 (between 200 and $575 \mathrm{~kW}$ ) - is due to the different individual efficiencies of the gyrotron response to the same applied voltage.

In this somewhat more delicate experiment, the best discrimination between the two beam absorption locations is obtained when the soft x-ray signals are not band-pass-filtered but only normalized along time, to ensure the highest signal dynamic. The linear fits are then performed between the power step-up and step-down times of the beams coming from launchers L1 and L5 separately, as indicated in figure 10, and an estimate of the power deposition location can be drawn for each beam at a $500 \mathrm{~Hz}$ rate, as indicated in figures 11 and 12 .

The expected trend of the evolution of both power deposition locations is recovered, but the detection of one beam is clearly disturbed by the heat pulses propagating from the other beam. The resulting power deposition locations are erroneously displaced toward each other.

The HRIM analysis is not shown for this experiment. But note that in the case of simultaneous amplitude modulation of several EC power beams (absorbed at different locations), the plasma response amplitude inferred from the HRIM analysis will reveal all the multiple deposition locations at once. Only the phase information can attribute each deposition location to the corresponding beam. 


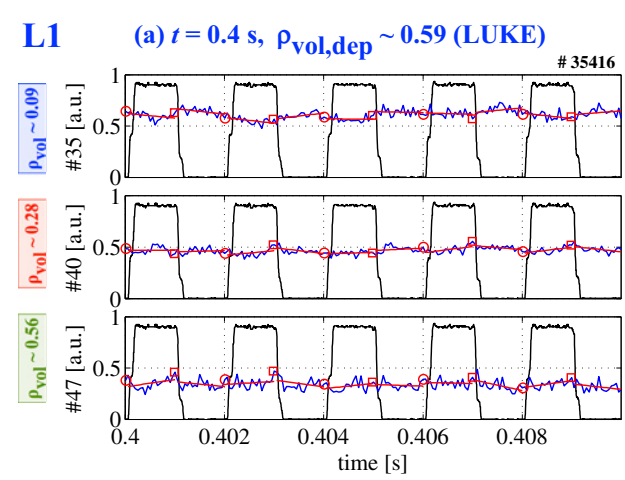

L5

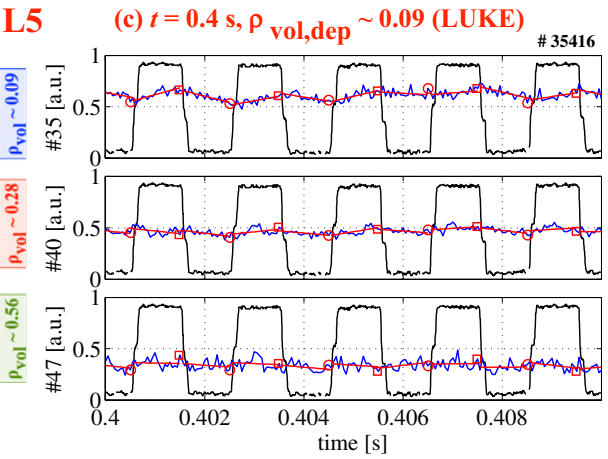

(b) $t=1.7 \mathrm{~s}, \rho_{\mathrm{vol}, \mathrm{dep}} \sim 0.07$ (LUKE)

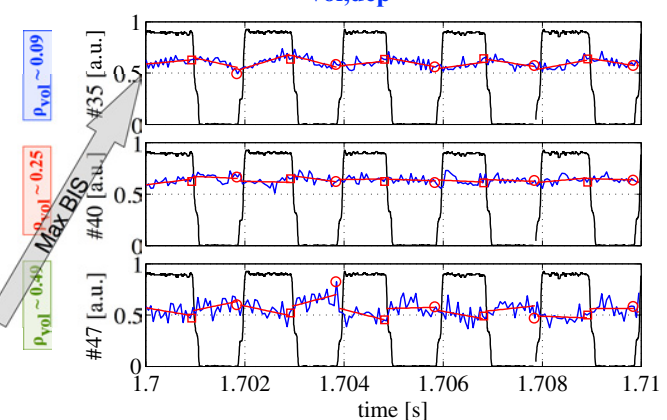

(d) $t=1.7 \mathrm{~s}, \rho_{\text {vol,dep }} \sim 0.59$ (LUKE)

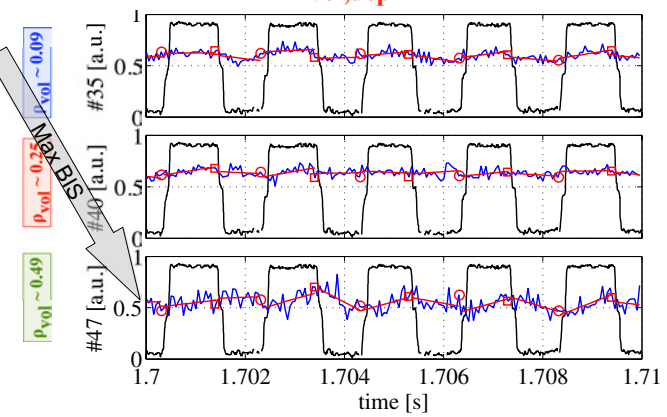

Figure 10. BIS analysis of the double beam case. BIS analysis of the soft x-ray signal from DMPX chords \#35, 40 and 47 at $(a),(c) t=0.4 \mathrm{~s}$ and $(b),(d) t=1.7 \mathrm{~s}$, for launchers $(a),(b) \mathrm{L} 1$ and $(c),(d)$ L5. The same normalized soft X-ray signals (in blue) are used for the BIS analysis of both beam power depositions. The linear fits (in red) are performed between the EC power step-up and step-down times (indicated by red circles and squares) of the X2 square waveforms (in black). Between $t=0.4 \mathrm{~s}$ and $t=1.7 \mathrm{~s}$, the maximum BIS amplitude has moved from the edge region to the plasma core and vice versa for the L1 and L5 launchers, respectively, as expected from the simulations in figure 9. TCV shot \#35416.

\section{Discussion}

In both the single and double beam experiments, the BIS analysis provides estimated power deposition profiles with a high time resolution (i.e. $f_{\text {mod }}=500 \mathrm{~Hz}$ or $2 f_{\text {mod }}=1 \mathrm{kHz}$ if both power step-up and step-down times are used).

In the single power deposition location tracking experiment, the experimental results inferred from the BIS analysis are in excellent agreement with the results obtained using the Fourier-like HRIM method applied on the same soft x-ray data. The BIS results are also in satisfactory agreement with the simulated power deposition location within $8.4 \%$ of $\rho_{\text {vol }}$, a systematic difference expected from line-integration effects. For central power deposition, fluctuations in the experimental results become large. This may be explained by the resurgence of a sawtooth activity when the X2 power is absorbed at the plasma center, which deteriorates the signal/noise ratio of the BIS analysis and jeopardizes the detection of the power deposition location.

In the double power deposition tracking experiment, the discrepancy between the BIS analysis results and the simulations is larger than that for the single beam experiment (up to $20 \%$ of $\rho_{\mathrm{vol}}$ ). Since the experiment and the simulation are in good agreement in the single 

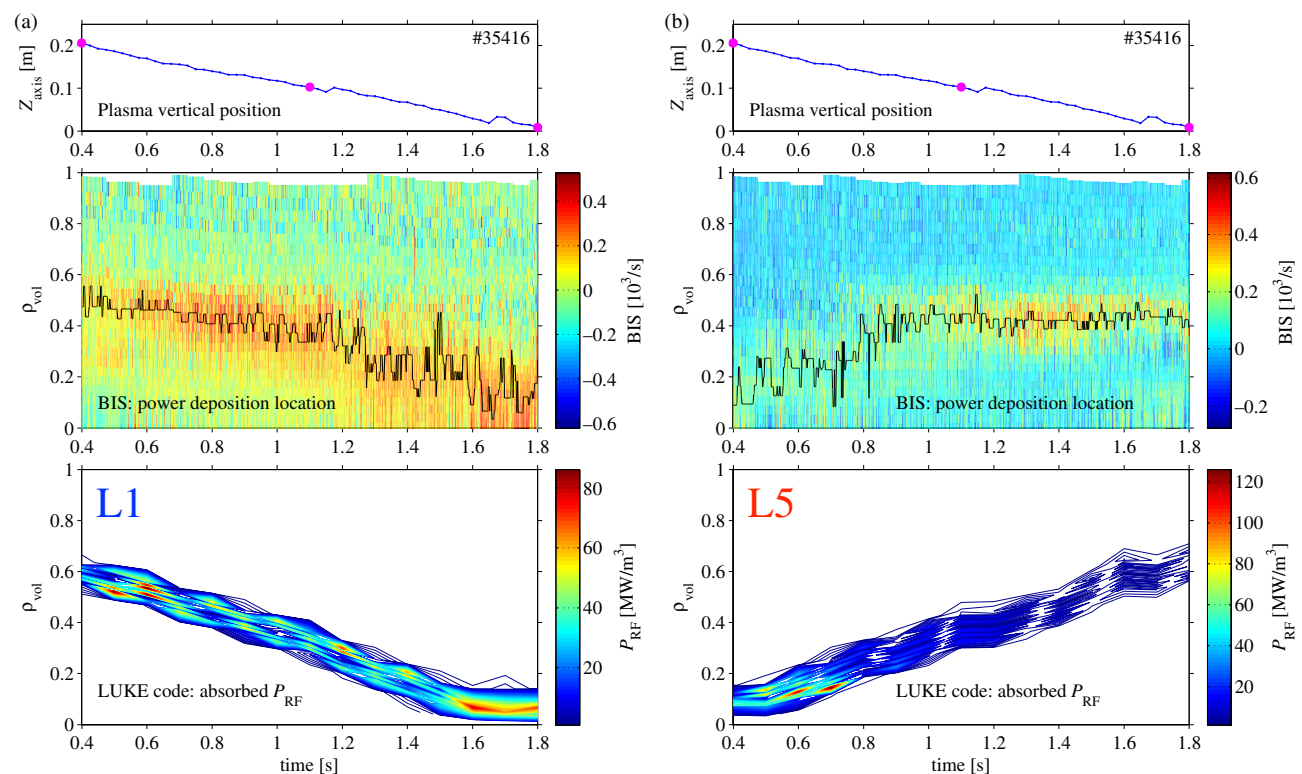

Figure 11. Simultaneous $X 2$ power deposition tracking. Same as in figure 6 but for the $X 2$ power beams injected from $(a)$ launcher L1 and $(b)$ launcher L5 simultaneously. The pink dots indicate the times corresponding to the C3PO simulations in figures 9(a)-(c). TCV shot \#35416.

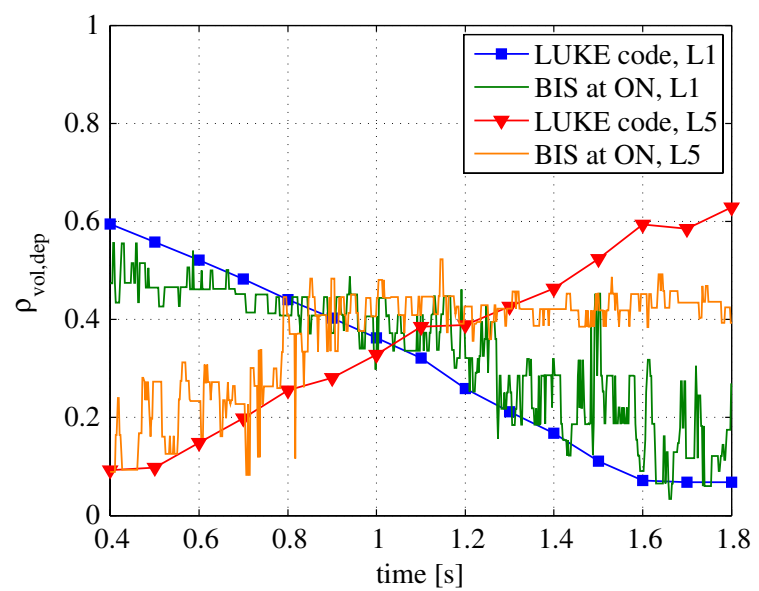

Figure 12. Simultaneous EC power deposition tracking. LUKE simulated power deposition locations for the X2 power beams injected from launcher L1 (blue $\mathbf{\square}$ ) and launcher L5 (red $\mathbf{\nabla}$ ), superimposed on the BIS estimated power deposition locations for L1 (green trace) and L5 (orange trace). TCV shot \#35416.

beam case (and that the temperature increase and relativistic effects are taken into account in the simulation through the experimental data input), the discrepancy in the double beam case can be attributed to a mutual disturbance of both beams in the BIS analysis of their respective absorption location: the power modulation of one beam perturbs the analysis of the response to the other beam modulation.

The double beam experiment was repeated with slower modulation frequencies (137 and $237 \mathrm{~Hz}$ instead of $500 \mathrm{~Hz}$ ) in order to improve the discrimination between the two beam 
deposition locations by performing the linear fits on quarters of the modulation period $\left(\tau_{\bmod } / 4\right.$ instead of $\tau_{\text {mod }} / 2$ ) around the power steps of one gyrotron so as to avoid the perturbation due to the power steps of the other gyrotron. But similar results were obtained and no clear improvement was observed.

\section{Conclusion}

Modulation of the amplitude of the injected auxiliary power is a common tool used to estimate the absorbed power profile in an experiment. Amongst the many methods proposed to analyze the plasma response to power modulation, the simple break-in-slope (BIS) analysis is the only one that provides an estimated power deposition profile at each power step-up and step-down time, i.e. at a high time rate. It has been successfully tested and compared with other methods in previous experiments under stationary conditions.

The present experiments make use of the unique flexibility of the TCV tokamak and of its EC power system to demonstrate the ability of the BIS analysis to track the EC power deposition location with a high time resolution in scenarios where the wave-plasma resonance location is moving during the discharge. The time-varying power deposition location of one $\mathrm{X} 2$ power beam is successfully tracked at a high time rate $(1 \mathrm{kHz})$ by BIS analysis of high temporal and radial resolutions soft $x$-ray profiles. The experimental BIS results are in excellent agreement with the results of a harmonic response identification HRIM analysis of the same soft $\mathrm{X}$-ray profiles and with the power absorption profiles simulated by the C3PO-LUKE code, except for the central deposition. The latter discrepancy may be caused by the resurgence of a periodic profile relaxation when the $\mathrm{X} 2 \mathrm{MECH}$ is deposited at the plasma center, as well as by line-integration effects which may become large in the plasma core where the profiles are flatter than in the plasma periphery.

Orthogonal power modulation waveforms allow us to track the power deposition locations of two X2 power beams simultaneously. The experimental results compare satisfactorily with the simulation, but the discrepancy is larger than in the single beam case due to the mutual disturbance of the two heating modulations.

The BIS analysis is thus a promising candidate for the detection and monitoring of the EC power deposition in real-time control applications.

\section{Acknowledgments}

This work was supported in part by the Swiss National Science Foundation. This work, supported by the European Communities under the contract of Association between EURATOM and CEA, was carried out within the framework of the European Fusion Development Agreement. The views and opinions expressed herein do not necessarily reflect those of the European Commission.

Euratom $@ 2011$.

\section{References}

[1] Angioni C et al 2003 Nucl. Fusion 43455

[2] Lennholm M et al 2009 Phys. Rev. Lett. 102115004

[3] Zohm H et al 2007 Nucl. Fusion 47228

[4] La Haye R J et al 2009 Nucl. Fusion 49045005

[5] Mueck A et al 2007 Phys. Rev. Lett. 98175004

[6] Manini A et al 2003 Nucl. Fusion 43490 
[7] Moret J-M et al 1993 Nucl. Fusion 331185

[8] Berrino B et al 2006 IEEE Trans. Nucl. Sci. 531009

[9] Jahns G L et al 1986 Nucl. Fusion 26226

[10] Hartfuss H J et al 1986 Nucl. Fusion 26678

[11] Paley J I et al 2009 Plasma Phys. Control. Fusion 51055010

[12] Gambier D J et al 1990 Nucl. Fusion 3023

[13] Van Eester D 2004 Plasma Phys. Control. Fusion 461675

[14] Lerche E A et al 2008 Plasma Phys. Control. Fusion 50035003

[15] Gasparino U et al 1988 Plasma Phys. Control. Fusion 30283

[16] Leuterer F et al 2003 Nucl. Fusion 43744

[17] Moret J-M 1988 PhD Thesis no 758, Ecole Polytechnique Fédérale de Lausanne (EPFL), CH-1015 Lausanne, Switzerland

[18] Hofmann F et al 1994 Plasma Phys. Control. Fusion 36 B277-B87

[19] Goodman T P et al 1996 Proc. 19th SOFT (Lisbon, Portugal) vol 1, p 565

[20] Behn R et al 1994 Proc. 21st EPS Conf. (Montpellier, France) vol 18B (ECA) p 1251

[21] Barry S. et al 1997 Proc. 8th LAPD Symp. (Doorwerth, Netherlands) p 313

[22] Hofmann F and Tonetti G 1988 Nucl. Fusion 281871

[23] Hofmann F. 1988 Comput. Phys. Commun. 48207

[24] Sushkov A et al 2008 Rev. Sci. Instrum. 79023506

[25] Hutchinson I H 2002 Principles of Plasma Diagnostics 2nd edn (Cambridge: Cambridge University Press)

[26] Decker J and Peysson Y 2004 Report EUR-CEA-FC-1736, Euratom-CEA

[27] Peysson Y and Decker J 2008 Report EUR-CEA-FC-1739, Euratom-CEA

[28] Simm C W 1987 PhD Thesis no 696, Ecole Polytechnique Fédérale de Lausanne (EPFL), CH-1015 Lausanne, Switzerland 